\title{
Gastric cancer increase the percentage of intermediate (CD14++CD16+) and nonclassical (CD14+CD16+) monocytes
}

\author{
ANDRZEJ ELJASZEWICZI, MICHAL JANKOWSKI ${ }^{\prime}$ LIDIA GACKOWSKA ${ }^{I}$, ANNA HELMIN-BASA ${ }^{\prime}$, \\ MALGORZATA WIESE ${ }^{l}$, IZABELA KUBISZEWSKA ${ }^{l}$, WOJCIECH KASZEWSKI', \\ JACEK MICHALKIEWICZ ${ }^{1,3}$, WOJCIECH ZEGARSKI
}

${ }^{1}$ Chair of Immunology, Collegium Medicum in Bydgoszcz, Nicolaus Copernicus University of Torun, Poland

${ }^{2}$ Department of Surgical Oncology, Collegium Medicum in Bydgoszcz, Nicoloaus Copernicus University of Torun, Poland ${ }^{3}$ Department of Microbiology and Immunology, Children's Memorial Hospital, Warsaw, Poland

\begin{abstract}
Introduction: Gastric cancer is one of the most common malignancies diagnosed worldwide. Despite therapeutic options, prognosis is unfavorable, particularly when dealing with an advanced stage carcinoma. Similarly to other malignancies, the development of gastric cancer is associated with increased macrophage infiltration. Monocytes are peripheral blood precursors of tissue macrophages. In the relation to the expression of $C D 14$ and CD16 receptors, human peripheral blood monocytes can be divided into: "clasical monocytes" CD14++CD16-, intermediate monocytes CD14++CD16+ and non-classical monocytes CD14+CD16+. However, little is known about monocytes involvement in the regulation of immune response in patients with gastric malignancies.

Material and methods: In this study, the phenotype of peripheral blood monocytes was determined in patients with gastric tumors by flow cytometry. We also analyzed the level of interleukin 10 (IL-10) and tumor necrosis factor $\alpha(T N F-\alpha)$ production after $24 \mathrm{~h}$ stimulation of peripheral blood mononuclear cells with lipopolysaccharide. The results obtained in group of gastric cancer patients were compared to those of age-matched group of healthy individuals.

Results: We observed a significant decrease in percentage of monocytes in the peripheral blood of gastric cancer patients as compared to healthy donors. Moreover gastric cancer patients shows a significant decrease of CD14++CD16-monocytes subpopulation and an increase in CD14++CD16+ and $C D 14+C D 16+$ monocytes subpopulations as compared to healthy volunteers. In stomach cancer patients we also have observed increased production of IL-10 as compared to controls.

Conclusions: In summary, this study has revealed that gastric malignancies significantly modulate the phenotype of peripheral blood monocytes giving them anti-inflammatory properties. Therapeutic targeting of anti-inflammatory monocytes in the peripheral blood could modulate the complex activities of macrophages in tumor environment.
\end{abstract}

Key words: gastric cancer, CD14++CD16-, CD14++CD16+, CD14+CD16+, IL-10, TNF- $\alpha$, flow cytometry.

(Centr Eur J Immunol 2012; 37 (4): 355-361)

\section{Introduction}

The growth of neoplastic tissue is associated with modulated activity and function of immune system, including monocytes and macrophages. Depending on the activating factor, monocytes and macrophages can be involved in a variety of biological processes, such as presentation of antigen, cytotoxicity, phagocytosis, secretion of biologi-

Correspondence: Andrzej Eljaszewicz, Chair of Immunology, Collegium Medicum in Bydgoszcz, Nicolaus Copernicus University, M. Sklodowskiej-Curie 9, 85-094 Bydgoszcz, Poland, e-mail: a.eljaszewicz@cm.umk.pl 
cally active factors, control of inflammatory processes, and rearrangement and reconstruction of destroyed tissues [1].

It is widely known that macrophages constitute the predominant component of leukocytic infiltrate in many malignancies including gastric tumors. They are referred to as tumor-associated macrophages (TAMs). Due to their pleiotropic biological properties, TAMs can influence the development of neoplastic tissue both progressively and regressively. Additionally, they control primary and secondary response of the immune system. Pro- and anti-neoplastic properties of TAMs are associated with their antiand pro-inflammatory activities, respectively, both determined by monocytes activating factors [2-6].

Monocytes are a population of cells that possess heterogenic morphology, phenotype and effector properties. Due to various levels of lipopolysaccharide (LPS) receptor (CD14) expression and the expression of receptor III for Fc $\gamma$ fragment (CD16), three subpopulations of monocytes can be distinguished: CD14++CD16-, CD14++CD16+, and CD14+CD16+. CD14++CD16- monocytes were defined as classical monocytes [7]. Under physiological conditions, these cells constitute from $85 \%$ to $95 \%$ of all peripheral monocytes. Remaining two subpopulations of monocytes showing high expression of CD16 differ in terms of CD14 expression levels and physiologically correspond up to $15 \%$ of peripheral blood monocytes [8,9]. Despite phenotypic similarity associated with the expression of CD16 receptor both aforementioned populations are characterized by different biological activities [10]. Increased fraction of CD14+CD16+ subpopulation amongst peripheral blood monocytes was observed in the course of infections and inflammatory processes $[11,12]$, in septic states [13], and in some malignancies $[14,15]$. The CD14+CD16+ cells are defined as nonclassical [7] monocytes since compared to classical monocytes, they synthesize and release relatively higher amounts of tumor necrosis factor $\alpha$ (TNF- $\alpha$ ) when stimulated with LPS, and do not secrete interleukin 10 (IL-10) or release only small amounts of this cytokine [16]. This population shows several similarities to tissue macrophages and similarly to the latter cells they are con-

Table 1. Characteristic of study populations

\begin{tabular}{|c|c|c|}
\hline & Patients & Control group \\
\hline$N$ & 33 & 17 \\
\hline Median age (range) & $62.79(30-86)$ & $58.35(49-68)$ \\
\hline Sex (woman/man) & $11 / 22$ & $8 / 9$ \\
\hline \multicolumn{3}{|c|}{ Stage (percentage of all studied patients) } \\
\hline I & $5(15.15 \%)$ & \\
\hline II & $2(6.06 \%)$ & \\
\hline III & $14(42.42 \%)$ & \\
\hline IV & $12(36.36 \%)$ & \\
\hline
\end{tabular}

sidered more mature cells than classical monocytes [17, 18]. Higher fraction of CD14++CD16+ monocytes was reported in neonatal septic states among others [19]. The presence of this cellular subpopulation in peripheral blood is interpreted as an intermediate stage of monocytes differentiation towards macrophages [10] and are referred to as intermediate monocytes [7]. Little is known about the phenotype of peripheral blood monocytes in patients with gastric malignancies. Moreover, previous observation of increasing the percentage of CD14+CD16+ monocytes in some malignancies $[14,15]$ were made based on the division of monocytes on CD14+CD16- and CD14+CD16+. Therefore, the aim of the present study was to determine the phenotype of peripheral blood monocytes in the gastric cancer patients in relation to the currently used classification of monocytes subpopulation described above. Additionally, we analyzed the levels of IL-10 and TNF- $\alpha$ produced after 24 hours LPS stimulation of peripheral blood mononuclear cells (PBMCs).

\section{Material and methods}

\section{Patients}

This study included gastric cancer patients treated at the Department of Oncological Surgery, Prof. F. Łukaszczyk Memorial Center of Oncology in Bydgoszcz (Poland). Thirty-three patients qualified to stomach resection were enrolled. None of the patients received chemotherapy and radiotherapy or was subjected to surgical intervention or blood transfusion six months prior the enrollment. Furthermore, none of the participants showed any clinical or cellular (lack of CD64 receptor expression on granulocytes) signs of ongoing infection. Following the stomach resection, surgical specimen was subjected to histopathologic examination (pTNM), in order to determine the stage of tumor according to the AJCC (American Joint Committee on Cancer). The results obtained in the patient group were compared to those of age-matched healthy individuals. Characteristics of patients and the controls are summarized in Table 1.

Peripheral blood was examined in all participants. The material was collected after receiving approval of the Bioethical Committee of the Collegium Medicum in Bydgoszcz. Each participant was familiarized with the objectives of the study and expressed written consent for material collection.

\section{Analysis of membrane receptors of monocytes}

Peripheral blood was labeled with murine anti-CD14PerCP (IgG2b; clone: MoP9, Becton Dickinson), murine anti-CD16-FITC (IgG1; clone NKP15, Becton Dickinson) and anti-CD64-PE (IgG1, clone: 10.1; Becton Dickinson), monoclonal antibodies. Isotype control was used for every staining, using the isotypes of antibodies included in the 
staining panel. The labeling was carried out following the manufacturer's instructions in accordance with the stainand-then-lyse technique. Briefly, $20 \mu \mathrm{l}$ of antibody suspension was added to $100 \mu 1$ of peripheral blood. The samples were mixed and incubated for 15-20 minutes in dark conditions at room temperature. Subsequently, $2 \mathrm{ml}$ of Lysing Solution (Becton Dickinson) was added to each sample and incubated for 15-20 minutes in darkness at room temperature. Then, $1 \mathrm{ml}$ of cold buffered saline (PBS, 2- $8^{\circ} \mathrm{C}$ ) was added to each sample and centrifuged $(300 \times \mathrm{g})$ for $10 \mathrm{~min}$ utes at $4^{\circ} \mathrm{C}$. After removing the supernatant, $2 \mathrm{ml}$ of PBS $\left(4^{\circ} \mathrm{C}\right)$ was added to the cellular sediment and again centrifuged $(300 \times \mathrm{g})$ for 10 minutes at $4^{\circ} \mathrm{C}$. After removing the supernatant, $200 \mu 1$ of PBS $\left(2-8^{\circ} \mathrm{C}\right)$ was added to the sediment and analyzed with the FACScan flow cytometer (Becton Dickinson). At least 40000 events were acquired.

Obtained cytometric data was analyzed using FlowJo version 7.6.1 (Tree Star). The analysis of the percentage of cells expressing studied receptors and/or their levels, expressed as the mean fluorescence intensity (MFI), was conducted in a population of cells characterized by medium-sized granules (SSCmed) and the expression of CD14 receptor - monocytes (Fig. 1).

\section{Isolation of peripheral blood mononuclear cells}

Peripheral blood mononuclear cells were isolated from heparinized whole blood by density gradient centrifugation (LSM 1072, PAA), performed following the manufacturer's instructions. Obtained suspension of PBMCs, containing $85-90 \%$ lymphocytes, 10-15\% monocytes, and approximately $1 \%$ granulocytes, was used for further stimulation. Cellular counts in suspension were determined using the Bürker's chamber. The viability of isolated cells exceeded $90 \%$ as confirmed using trypan blue [20].

\section{In vitro stimulation}

Peripheral blood mononuclear cells were subjected to 24-hour stimulation with Escherichia coli LPS (SigmaAldrich). The cells were cultured in $1 \mathrm{ml}$ of culture medium, in sterile non-adherent polypropylene tubes (Falcon, Becton Dickinson). Each sample contained $1 \times 10^{6}$ of PBMCs. The cells were stimulated with $1 \mu \mathrm{g}$ of E. coli LPS (experimental sample) or left non-stimulated (control sample), and cultured for 24 hours under standard conditions $\left(37^{\circ} \mathrm{C}, 5 \% \mathrm{CO}_{2}\right)$.

\section{Determination of cytokines concentration by means of immunoenzymatic ELISA method}

Concentrations of cytokines (IL-10 and TNF- $\alpha$ ) in the supernatants of PBMCs cultures subjected to 24-stimulation were determined by means of immunoenzymatic ELISA tests (Becton Dickinson). The sensitivity range of both ELISA tests was the same, ranging from 7.8 to $500 \mathrm{pg} / \mathrm{ml}$. The samples were analyzed with automated light absorbance

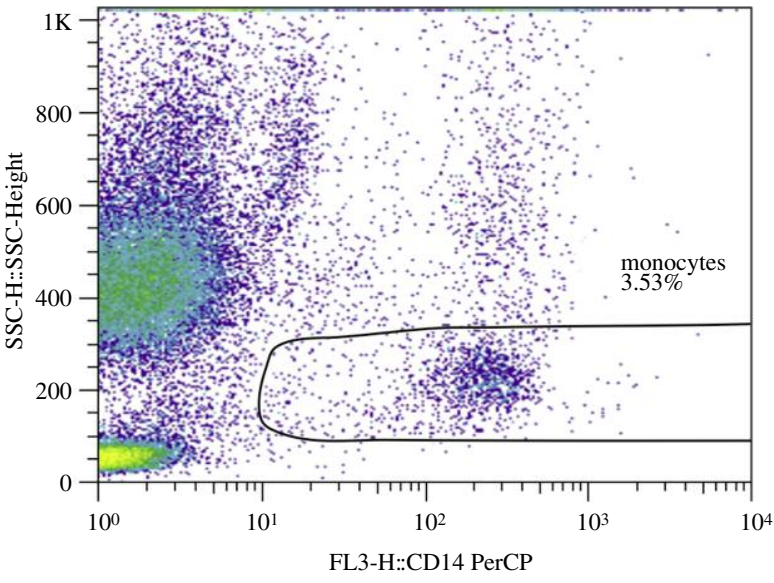

Fig. 1. Gaiting of human peripheral blood monocytes. Monocytes were referred to as CD14+ and SSC med cells

reader iEMS Reader MF (Labsystems) at $450 \mathrm{~nm}$ wavelength. The contents of analyzed cytokines were calculated by Genesis (version 2.2.) software, enabling the expression of the results as an absolute concentration $(\mathrm{pg} / \mathrm{ml})$.

\section{Statistical analysis}

Statistical analysis of obtained results was carried out using Statistica version 9 statistical package (StatSoft Inc.). The normality of distribution of all analyzed variables was verified with the Shapiro-Wilk W test. Based on the results of this test, the following tests were used for data analysis: a) Student's $t$ test - for normally distributed variables with homogeneity of variance confirmed by the Levene's test (results were expressed as arithmetic means \pm SD); b) Mann-Whitney $U$ test - for variables whose distributions differed from normal in at least one of compared groups (results were expressed as medians and ranges). The differences were considered significant at $p<0.05$.

\section{Results}

\section{Distribution of peripheral blood monocytes and their subpopulations}

A significant decrease $(p=0.0139)$ in a fraction of monocytes (CD14+/SSCmed) was observed in the population of blood leukocytes obtained from patients with gastric malignancies $(3.25 \pm 1.25 ; n=33)$ as compared to the control group (4.34 $\pm 1.74 ; n=17$ ) (Fig. 2). Additionally, there was a tendency observed $(p=0.0824)$ for a decreased expression of CD14 receptor, expressed as a mean fluorescence intensity (MFI), on CD14-positive cells of the patients (171.87 $\pm 51.29)$ as compared to the controls $(200.12 \pm 55.17)$. Analyzing the distribution of basic subpopulations of peripheral blood monocytes, we observed a significant decrease in 

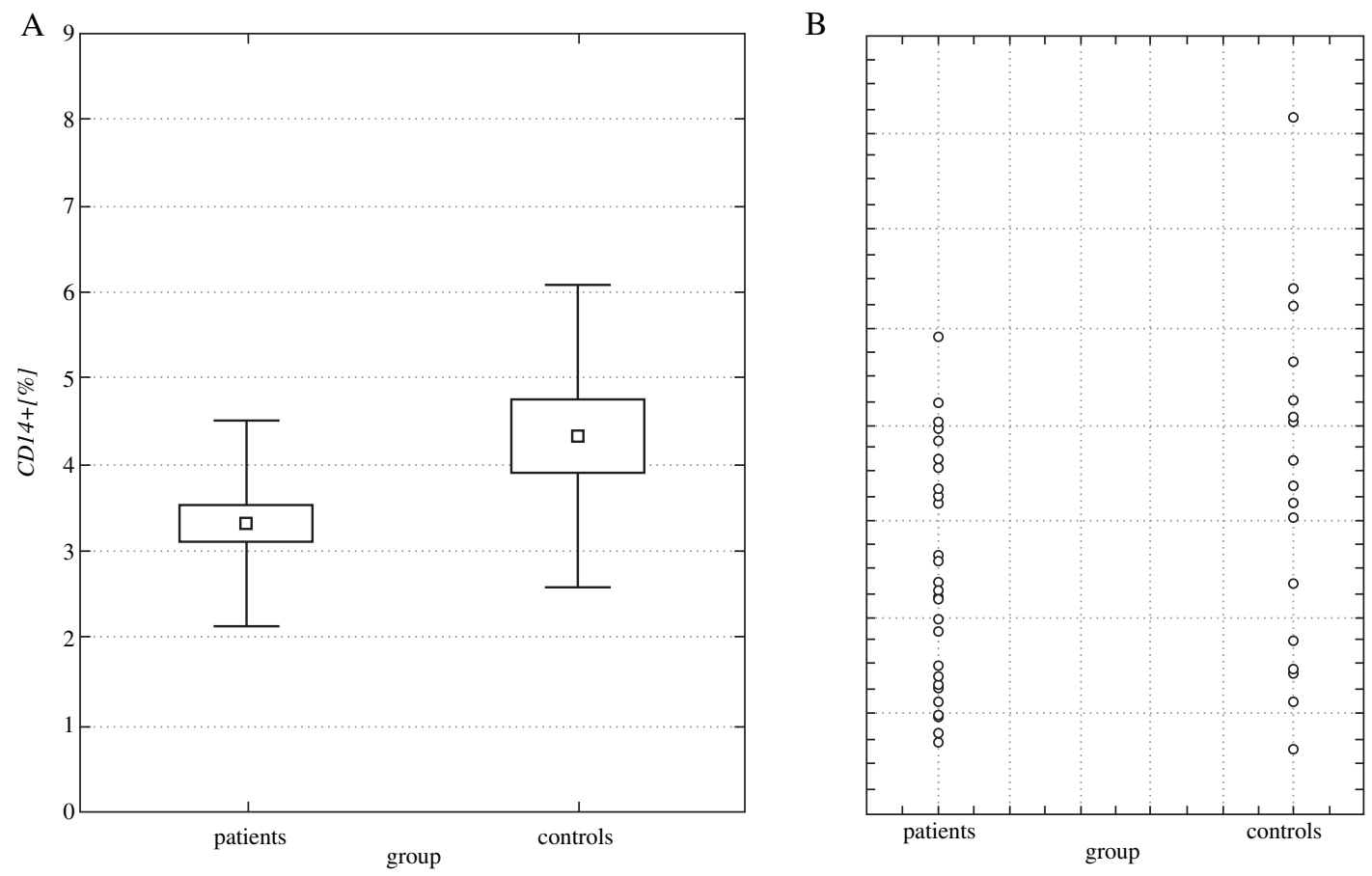

Fig. 2. Distribution of peripheral blood monocytes. A) Box-and-whisker plot show: the mean $(\bar{x})$; the standard error of the mean (box); the mean $\pm \mathrm{SD}$ (whiskers). B) Scatter plot of raw data. CD14+ [\%]- percentage of monocytes; patients $n=34$; controls $n=17$

the fraction of so-called classic monocytes (CD14++CD16-; $p<0.0001)$, and an increase in CD14++CD16+ $(p<0.0001)$ and CD14+CD16+ $(p=0.0003)$ monocytes in gastric cancer patients as compared to the healthy volunteers (Fig. 3).

\section{Levels of IL-10 and TNF- $\alpha$ in culture supernatants of PBMCs after 24-hour stimulation}

After 24-hour stimulation of PBMCs with LPS, a significant increase in IL-10 concentration $(p=0.0194)$ was observed in gastric cancer patients as compared to healthy individuals. Additionally, a tendency was observed $(p=0.0712)$ for lower TNF- $\alpha$ levels in the experimental group as compared to the controls (Table 2).

\section{Discussion}

Monocytes are cells of myeloid origin, which circulate in the peripheral blood up to 72 hours. They undergo apoptosis if they are not stimulated by a signal causing their activation and/or inducing their migration to the site of ongoing immune response. In contrast, activated monocytes migrate to tissues where they transform into macrophages [7], that can further differentiate into myeloid dendritic cells under favorable conditions [21, 22]. A decrease in the fraction of monocytes in the pool of peripheral leukocytes in patients with gastric malignancies that was observed in this study probably suggests an enhanced migration of these

Table 2. The level of IL-10 and TNF- $\alpha$ secretion after $24 \mathrm{~h}$ stimulation of PBMCs

\begin{tabular}{|c|c|c|c|}
\hline \multirow[t]{2}{*}{ Cytokine } & \multicolumn{2}{|c|}{ Group } & \multirow[t]{2}{*}{$p$ value } \\
\hline & $\begin{array}{l}\text { Patients } \\
(n=21)\end{array}$ & $\begin{array}{l}\text { Control group } \\
\qquad(n=15)\end{array}$ & \\
\hline IL-10 & $702.42[100.99 ; 35463] \uparrow$ & $128.58[102.14 ; 2380.813]$ & 0.0194 \\
\hline TNF- $\alpha$ & $188.99[101.74 ; 2626.53] \downarrow$ & $460.72[362.59 ; 963.14]$ & 0.0712 \\
\hline
\end{tabular}

The levels of IL-10 and TNF- $\alpha$ were measured in culture supernatants of PBMCs stimulated with E. coli LPS for 24 hours, and in non-stimulated controls. In order to eliminate the background effects of spontaneous cytokine synthesis, the results were expressed as a percentage of cytokine concentration in a supernatant of control samples (considered as 100\%); Mann-Whitney U test was used, results are presented as medians [min; max] 
A

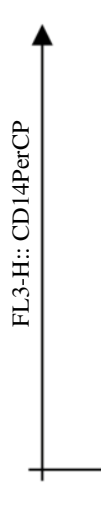

Patient

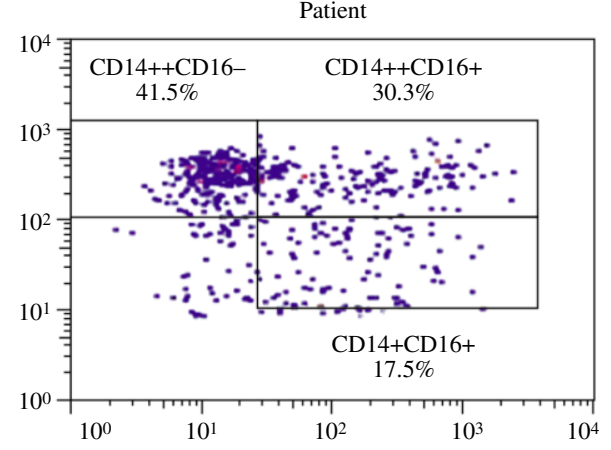

Control

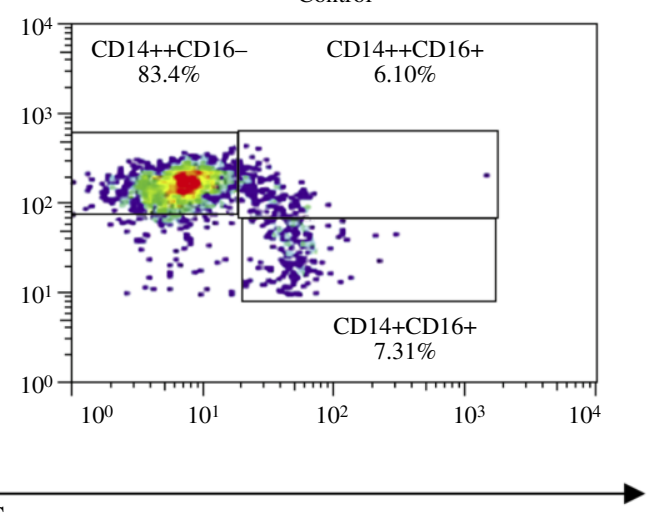

FL1-H:: CD16 FITC

B

\begin{tabular}{|cccc|}
\hline $\begin{array}{c}\text { Monocytes } \\
\text { subpopulations }\end{array}$ & $\begin{array}{c}\text { Patients } \\
(\boldsymbol{n}=\mathbf{3 3})\end{array}$ & $\begin{array}{c}\text { Control group } \\
(\boldsymbol{n}=\mathbf{1 7})\end{array}$ & $\boldsymbol{p}$ value \\
\hline $\begin{array}{c}\text { Classical } \\
(\mathrm{CD} 14++\mathrm{CD} 16-)\end{array}$ & $56.32(2.44 ; 90.20)$ & $87.90(81.46 ; 94.10)$ & $<0.0001$ \\
\hline $\begin{array}{c}\text { Intermediate } \\
(\mathrm{CD} 14++\mathrm{CD} 16+)\end{array}$ & $25.07(3.50 ; 84.10)$ & $3.27(1.97 ; 10.56)$ & $<0.0001$ \\
\hline $\begin{array}{c}\text { Nonclassical } \\
(\mathrm{CD} 14+\mathrm{CD} 16+)\end{array}$ & $11.20(30.05 ; 46.07)$ & $5.06(2.41 ; 9.76)$ & $<0.0003$ \\
\hline
\end{tabular}

Fig. 3. Distribution of peripheral blood monocytes subpopulations. A - dot plots of representative data of both analyzed groups; $\mathrm{B}$ - the percentage of peripheral blood monocytes subpopulations in the analyzed groups. $\%$ - percentage of positive cells; Mann-Whitney $U$ test was used. Results are presented as medians [min; max]

cells to the site of tumor tissue. There, as tumor-associated macrophages, they can affect tumor development both positively and negatively. Pro- or anti-neoplastic activity of TAMs is associated with their polarization towards antiinflammatory (macrophages with MII phenotype) or proinflammatory cells (macrophages with MI phenotype), respectively [23]. In the case of gastric malignancies, these activities differ depending on the region of the tumor. Histopathologically, three regions of gastric tumor can be distinguished: a) tumor nests - aggregations of neoplastic cells clearly distinguishable from necrotic foci and the stromal components; b) tumor center - a region with numerous necrotic foci, and c) tumor margin - intermediate zone between the invasive front of malignancy and surrounding healthy tissue. Macrophages in the tumor nest show a variety of pro-inflammatory activities, such as those leading to malignant cell apoptosis and enhanced infiltration of cytotoxic T lymphocytes (CTL). However, one should be aware that the number of TAMs in tumor nest is relatively small as compared to the remaining two tumor regions. In contrast to tumor nest, macrophages infiltrating tumor margin and its center are postulated to show the activities of antiinflammatory cells, involved in the processes of angiogenesis, among others [2]. Differentiation of peripheral blood monocytes towards macrophages is associated with the presence of three consecutive subpopulations [8]. They can be distinguished by flow cytometry based on differences in CD14 expression levels and the presence of CD16 receptor. Consequently, decreased fraction of classical monocytes observed in this study along with simultaneous increase in the remaining two subpopulations, has confirmed the hypothesis that these cells differentiate more rapidly into tissue macrophages. M-CSF is a factor that can potentially intensify the kinetics of peripheral blood monocytes, and its high concentrations were reported from gastric cancer patients [24]. Administration of exogenous M-CSF to healthy individuals induces formation of CD16+ monocytes that phenotypically are similar to those induced spontaneously in patients with gastric malignancies [15]. Noticeably, monocytes subpopulations differ in terms of their degree of biological activities, such as the profile of released cytokines, phagocytic and cytotoxic properties, and the ability to present antigen to naive $\mathrm{T}$ lymphocytes [10]. The CD14+CD16+ monocytes are considered pro-inflammatory cells, since following stimulation with LPS they synthesize high amounts of TNF- $\alpha$ and small amounts of IL-10, if any [16]. In contrast, the cells with CD14++CD16+ phenotype synthesize high amounts of IL-10, which suggests 
their anti-inflammatory potential [10]. Thus, there is functional similarity between the subpopulations of monocytes showing the expression of CD16 receptor and tissue macrophages. In gastric malignancies, particularly in more advanced stages of this disease, peripheral blood monocytes show elevated ability for synthesis of IL-10. High plasma concentrations of IL-10 promote monocyte differentiation to macrophages, but inhibit their differentiation to dendritic cells [25]. Strong anti-inflammatory signal in association with the action of chemotactic factors is reflected by monocyte differentiation to MII macrophages, as a result promoting the growth of neoplastic tissue. Monocytes with CD14+CD16+ phenotype show the highest anti-neoplastic activity in vitro, among others exerting cytotoxic and cytostatic effects on malignant cells, as well as synthesizing and secreting high amounts of pro-inflammatory cytokines (TNF- $\alpha$, IL-12p40/p70) [26]. The observed increase in the fraction of monocytes from this subpopulation, in peripheral blood of patients, is undoubtedly associated with the immune system response against malignant cells circulating in the peripheral blood. On the other hand, the involvement of pro-inflammatory monocytes at the site of neoplastic tissue growth can explain the anti-neoplastic activity of the MI macrophages infiltrating tumor nests [27].

The results of previous studies suggest high potential for spontaneous synthesis of IL-10 by peripheral blood monocytes in patients with advanced gastric malignancies [28]. Presentation of results as a percentage of control is intended to show the actual level of IL-10 and TNF- $\alpha$ secretion in response to $E$. coli LPS stimulation. Using this approach, we have revealed in this study that mononuclear cells in the peripheral blood of patients with gastric malignancies are characterized by enhanced secretion of IL-10 in response to LPS stimulation as compared to healthy individuals. As previously mentioned, this study revealed a trend (at a threshold of statistical significance) towards decreased expression of CD14 receptor on the monocytes of patients with gastric malignancies. This finding can be associated with a dysfunction of these cells in response to LPS, leading to decreased synthesis and secretion of TNF- $\alpha$ (also at a threshold of statistical significance). It should be noted; however, that signal transmission from monocyte cellular membrane, leading to their activation in response to LPS stimulation, is mediated by the Toll like receptor 4 (TLR-4) [29]. We did not observed significant differences in the fraction of cells showing expression of this receptor or the expression level (data not shown). Consequently it can be assumed that monocytes of gastric cancer patients are characterized by normal ability to respond to bacterial toxin stimulation. Thus, significant increase in IL-10 level observed in supernatant of LPS-stimulated cultures can be associated with higher fraction of CD14++CD16+ monocytes. As previously mentioned, this subpopulation of monocytes constitutes the principal source of IL-10 released in response to LPS stimulation [10]. Those observations suggest that tumor growth causes the polarization of monocytes/macrophages into anti-inflammatory cells already in the peripheral blood. Therapeutic targeting of monocytes in the peripheral blood could modulate the complex activities of macrophages in tumor environment.

No company had any input into or influence on the design, analyses, interpretation, or content of this manuscript. There are no conflicts of interest for any author.

This study was partially supported by the promotor grant from the Nicolaus Copernicus University (no. 47/2009), PhD advancement scholarship from EFS.

\section{References}

1. Gordon S, Taylor PR (2005): Monocyte and macrophage heterogeneity. Nat Rev Immunol 5: 953-964.

2. Ohno S, Inagawa H, Dhar DK, et al. (2003): The degree of macrophage infiltration into the cancer cell nest is a significant predictor of survival in gastric cancer patients. Anticancer Res 23: 5015-5022.

3. Leek RD, Lewis CE, Whitehouse R, et al. (1996): Association of macrophage infiltration with angiogenesis and prognosis in invasive breast carcinoma. Cancer Res 1996; 56: 4625-4629.

4. Shimura S, Yang G, Ebara S, et al. (2000): Reduced infiltration of tumor-associated macrophages in human prostate cancer: association with cancer progression. Cancer Res 60: 5857-5861.

5. Eljaszewicz A, Gackowska L, Kubiszewska I, et al. (2010): Macrophage activity in tumour development. Wspolczesna Onkol 14: 1-6.

6. Szaflarska A, Siedlar M, Zembala M (2003): Human monocytes/macrophages in the antitumour response of the host. Centr Eur J Immunol 28: 88-103.

7. Ziegler-Heitbrock L, Ancuta P, Crowe S, et al. (2010): Nomenclature of monocytes and dendritic cells in blood. Blood 116: e74-80.

8. Ziegler-Heitbrock HW (1996): Heterogeneity of human blood monocytes: the CD14+ CD16+ subpopulation. Immunol Today 17: 424-428.

9. Grage-Griebenow E, Flad HD, Ernst M (2001): Heterogeneity of human peripheral blood monocyte subsets. J Leukoc Biol 69: 11-20.

10. Skrzeczynska-Moncznik J, Bzowska M, Loseke S, et al. (2008): Peripheral blood CD14high CD16+ monocytes are main producers of IL-10. Scand J Immunol 67: 152-159.

11. Nockher WA, Scherberich JE (1998): Expanded CD14+ CD16+ monocyte subpopulation in patients with acute and chronic infections undergoing hemodialysis. Infect Immun 66: 2782-2790.

12. Katayama K, Matsubara T, Fujiwara M, et al. (2000): CD14+CD16+ monocyte subpopulation in Kawasaki disease. Clin Exp Immunol 121: 566-570.

13. Fingerle G, Pforte A, Passlick B, et al. (1993): The novel subset of CD14+/CD16+ blood monocytes is expanded in sepsis patients. Blood 82: 3170-3176.

14. Feng AL, Zhu JK, Sun JT, et al. (2011): CD16+ monocytes in breast cancer patients: expanded by monocyte chemoattractant protein-1 and may be useful for early diagnosis. Clin Exp Immunol 164: 57-65. 
15. Saleh MN, Goldman SJ, LoBuglio AF, et al. (1995): CD16+ monocytes in patients with cancer: spontaneous elevation and pharmacologic induction by recombinant human macrophage colony-stimulating factor. Blood 85: 2910-2917.

16. Belge KU, Dayyani F, Horelt A, et al. (2002): The proinflammatory $\mathrm{CD} 14+\mathrm{CD} 16+\mathrm{DR}++$ monocytes are a major source of TNF. J Immunol 168: 3536-3542.

17. Ziegler-Heitbrock L (2007): The CD14+ CD16+ blood monocytes: their role in infection and inflammation. J Leukoc Biol 81: 584-592.

18. Rivier A, Pčne J, Rabesandratana H, et al. (1995): Blood monocytes of untreated asthmatics exhibit some features of tissue macrophages. Clin Exp Immunol 100: 314-318.

19. Skrzeczyńska J, Kobylarz K, Hartwich Z, et al. (2002): CD14+CD16+ monocytes in the course of sepsis in neonates and small children: monitoring and functional studies. Scand J Immunol 55: 629-638.

20. Michalkiewicz J, Stachowski J, Barth C, et al. (1998): Effect of Pseudomonas aeruginosa exotoxin A on CD3-induced human T-cell activation. Immunol Lett 61: 79-88.

21. Peters JH, Ruppert J, Gieseler RK, et al. (1991): Differentiation of human monocytes into CD14 negative accessory cells: do dendritic cells derive from the monocytic lineage? Pathobiology 59: 122-126.

22. Randolph GJ, Inaba K, Robbiani DF, et al. (1999): Differentiation of phagocytic monocytes into lymph node dendritic cells in vivo. Immunity 11: 753-761.

23. Mantovani A, Sozzani S, Locati M, et al. (2002): Macrophage polarization: tumor-associated macrophages as a paradigm for polarized M2 mononuclear phagocytes. Trends Immunol 23: 549-555.

24. Mroczko B, Wereszczyńska-Siemiatkowska U, Groblewska M, et al. (2006): The diagnostic value of hematopoietic cytokines measurement in the sera of gastric cancer and gastric ulcer patients. Clin Chim Acta 374: 165-167.

25. Allavena P, Piemonti L, Longoni D, et al. (1998): IL-10 prevents the differentiation of monocytes to dendritic cells but promotes their maturation to macrophages. Eur J Immunol 28: 359-369.

26. Szaflarska A, Baj-Krzyworzeka M, Siedlar M, et al. (2004): Antitumor response of CD14+/CD16+ monocyte subpopulation. Exp Hematol 32: 748-755.

27. Aderem A, Underhill DM (1999): Mechanisms of phagocytosis in macrophages. Annu Rev Immunol 17: 593-623.

28. Sugai H, Kono K, Takahashi A, et al. (2004): Characteristic alteration of monocytes with increased intracellular IL-10 and IL-12 in patients with advanced-stage gastric cancer. J Surg Res 116: 277-287.

29. Tamandl D, Bahrami M, Wessner B, et al. (2003): Modulation of toll-like receptor 4 expression on human monocytes by tumor necrosis factor and interleukin-6: tumor necrosis factor evokes lipopolysaccharide hyporesponsiveness, whereas interleukin-6 enhances lipopolysaccharide activity. Shock 20: 224-229. 